BULLETIN OF THE

AMERICAN MATHEMATICAL SOCIETY

Volume 78, Number 5, September 1972

\title{
A THULLEN TYPE EXTENSION THEOREM FOR POSITIVE HOLOMORPHIC VECTOR BUNDLES ${ }^{1}$
}

\author{
BY YUM-TONG SIU ${ }^{2}$ \\ Communicated by S. S. Chern, April 3, 1972
}

We announce the following result.

THEOREM. Suppose $X$ is a complex manifold, $A$ is an analytic subset of $X$ of codimension $\geqq 1$, and $G$ is an open subset of $X$ which intersects every branch of $A$ of codimension 1. Suppose $V$ is a semipositive holomorphic vector bundle over $(X-A) \cup G$ (i.e. $V$ carries a hermitian metric with positive semidefinite curvature form). Then the sheaf $\mathcal{O}(V)$ of germs of holomorphic sections of $V$ can be extended uniquely to a reflexive coherent analytic sheaf over $X$.

COROLlary. If $\operatorname{dim} X=2$, then $V$ can be extended uniquely to a holomorphic vector bundle over $X$.

The special case where $A$ has codimension $\geqq 2$ and $V$ is a line bundle was proved by Shiffman [3], [4]. An alternative proof of Shiffman's line bundle result was given by Harvey [1] whose proof works also when $A$ is an arbitrary closed subset of $X$ with Hausdorff $(2 \operatorname{dim} X-3)$ measure 0 .

Our Corollary implies a theorem of Thullen [6, Satz 2], because, in a special case general enough to give the general case, the line bundle associated to the analytic subset of codimension 1 which is to be extended is semipositive.

The proof of our Theorem follows from Hörmander's $L^{2}$ estimates for the $\bar{\partial}$ operator [2] and the easy part of the usual sheaf-extension techniques (see e.g. [5] and related papers listed in the bibliography there). Let $\Delta_{r}=\{z \in C|| z \mid<r\}$ and $\Delta=\Delta_{1}$. We outline here the proof of our Theorem for the special case where $X=\Delta \times \Delta, A=\Delta \times\{0\}$, and $G=\Delta_{1 / 2} \times \Delta$.

Fix arbitrarily $\frac{1}{2}<r<1$. Let $f_{1}, \ldots, f_{k}$ be holomorphic sections of $V$ over $\Delta \times(\Delta-\{0\})$ generating $\mathcal{O}(V)$ there. Take arbitrarily $c \in \Delta-\{0\}$. Let $\rho=\rho\left(z_{2}\right)$ be a $C^{\infty}$ function on $\Delta-\{0\}$ with compact support such that $\rho \equiv 1$ on a neighborhood of $c$. Since $\left(z_{2}-c\right)^{-1} \bar{\partial}\left(\rho f_{j}\right) \mid \Delta_{r} \times(\Delta-\{0\})$ has

AMS 1969 subject classifications. Primary 3227, 3250; Secondary 3519.

Key words and phrases. Positive vector bundle, curvature form, coherent sheaf extension, differential operator estimates.

${ }^{1}$ Research partially supported by National Science Foundation Grant GP-27624.

2 Sloan Fellow. 
finite $L^{2}$-norm with respect to the given metric $h$ of $V$, by Hörmander's method we can find a $C^{\infty}$ section $g_{j}$ of $V$ over $\Delta_{r} \times(\Delta-\{0\})$ such that $g_{j}$ has finite $L^{2}$-norm with respect to $h$ and $\bar{\partial} g_{j}=\left(z_{2}-c\right)^{-1} \bar{\partial}\left(\rho f_{j}\right)$. It is well known that a holomorphic function defined outside an analytic subset of codimension $\geqq 1$ can be extended across it if the function is locally $L^{2}$ at every point of the analytic subset. Hence $\rho f_{j}-\left(z_{2}-c\right) g_{j}$ can be extended to a holomorphic section $s_{j}$ of $V$ over $\left(\Delta_{r} \times(\Delta-\{0\})\right)$ $\cup G$. The sections $s_{1}, \ldots, s_{k}$ generate $\mathcal{O}(V)$ at $\Delta_{r} \times\{c\}$. Likewise we can find holomorphic sections of $V$ over $\left(\Delta \times\left(\Delta_{r}-\{0\}\right)\right) \cup\left(\Delta_{1 / 2} \times \Delta_{r}\right)$ generating $\mathcal{O}(V)$ at $\Delta_{1 / 2} \times\{0\}$. The Theorem-for this case now follows from wellknown easy sheaf-extension techniques.

Theorems on extending semipositive holomorphic vector bundles across closed subsets with Hausdorff measure conditions can also be obtained.

Details will appear elsewhere.

\section{REFERENCES}

1. F. R. Harvey, A result on extending positive currents, preprint 1971.

2. L. Hörmander, $L^{2}$ estimates and existence theorems for the $\bar{\partial}$ operator, Acta Math. 113 (1965), 89-152. MR 31 \#3691.

3. B. Shiffman, Extension of positive holomorphic line bundles, Bull. Amer. Math. Soc. 77 (1971), 1091-1093.

4. , Extension of positive line bundles and meromorphic maps, Invent. Math. 15 (1972), 332-347.

5. Y.-T. Siu, A Hartogs type extension theorem for coherent analytic sheaves, Ann. of Math. (2) $93(1971), 166-188$.

6. P. Thullen, Über die wesentlichen Singularitäten analytischer Funktionen und Flächen im Raume von n komplexen Veränderlichen, Math. Ann. 111 (1935), 137-157.

Department of Mathematics, Yale University, New Haven, Connecticut 06520 